\title{
Clinical characteristics of keratoconus patients at the University of KwaZulu-Natal eye clinic
}

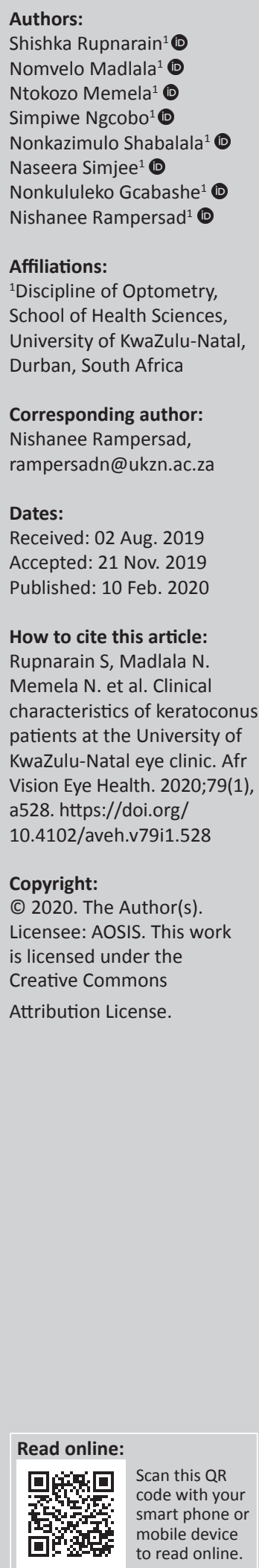

Background: Patients with keratoconus, which is a common corneal ectasia, often present to specialised clinics for management. Understanding the clinical characteristics of keratoconus patients can help improve knowledge of the presentation and management of this corneal ectasia and predict the needs of the clinic providing care for affected individuals.

Aim: To describe the clinical characteristics of keratoconus patients attending the University of KwaZulu-Natal (UKZN) eye clinic.

Setting: University of KwaZulu-Natal eye clinic.

Methods: The study used a retrospective research design by reviewing the clinical record cards of patients attending the UKZN contact lens eye clinic over a 4-year period (January 2014 to December 2017). Data related to age, clinical characteristics and method of management of the keratoconus patients were extracted and analysed using descriptive statistics.

Results: Just less than one-quarter of all patients $(n=1210)$ attending the UKZN contact lens eye clinic had keratoconus that was most often bilateral. The mean age at presentation was $25.2 \pm 9.6$ years with $74 \%$ of the sample being younger than 30 years. More than $90 \%(n=419)$ of the sample reported refractive reasons as the primary reason for presenting to the clinic. The majority of the sample had severe keratoconus $(n=257)$ and rigid contact lenses were most commonly used for management of keratoconus patients.

Conclusion: Keratoconus presents at an early age with a more severe grade and it is most commonly managed using rigid contact lenses. These findings should be considered for keratoconus screening, diagnosis and treatment programmes in KwaZulu-Natal.

Keywords: keratoconus; clinical characteristics; eye clinic; retrospective review; contact lenses.

\section{Introduction}

Keratoconus is a common progressive corneal ectasia characterised by corneal thinning and protrusion that leads to the development of myopia and irregular astigmatism. ${ }^{1-3}$ Consequently, patients with keratoconus frequently complain of blurred and distorted vision, halos and/or frequent changes in their spectacle prescriptions. ${ }^{2,4,5}$ In the early stage of keratoconus, spectacles and sometimes soft contact lenses are often used to correct the caused refractive error. ${ }^{6,7}$ However, as this corneal ectasia progresses, the irregular astigmatism that results is better managed with the use of rigid (corneal and scleral) contact lenses as they serve to provide a uniform anterior ocular surface and consequently improve vision. ${ }^{8}$ Surgery is also considered in the management of keratoconus and this includes collagen cross-linking and intra-stromal ring segment implants. Penetrating keratoplasty is advised for the advanced stage of keratoconus when there is contact lens intolerance, poor fitting of contact lenses, sub-optimal visual outcomes and/or frequent displacement of the rigid contact lenses. ${ }^{2,4}$

Data related to the incidence and prevalence estimates of keratoconus vary in different populations worldwide, and this may be attributed to the varying diagnostic criteria, definitions and methods of investigation that have been used in different studies. ${ }^{3}$ An early study reported the incidence of keratoconus in the general population to be between 50 and 230 per 100000 individuals with a prevalence of 54.5 per 100000 individuals. ${ }^{1}$ Even though the exact aetiology of keratoconus is unknown, certain factors have been associated with this corneal ectasia. Some studies have reported a link between excessive eye rubbing and atopy with keratoconus. ${ }^{3,9,10}$ It is also suggested that the incidence of keratoconus is higher in countries that have hot, dry and dusty climates. ${ }^{8}$ Despite being largely an isolated condition, keratoconus may be associated with certain systemic 
(Down's syndrome and Marfan's syndrome) and ocular (retinitis pigmentosa, aniridia and vernal keratoconjunctivitis) conditions., ${ }^{2,11}$ Although an early study ${ }^{1}$ reported that keratoconus affects all ethnic groups similarly, some researchers have shown differences between Asian and Caucasian individuals ${ }^{12}$ as well as the different Asian subpopulations, ${ }^{13}$ suggesting that genetic and/or environmental factors may have an influence on the development and progression of keratoconus.

The clinical characteristics of patients with keratoconus have been reported from several non-African countries. ${ }^{9-11,14,15}$ In contrast, there are only a few studies that have reported on keratoconus patients in Africa. ${ }^{16,17}$ Overall, these studies have reported on characteristics such as age, gender, corneal power, severity of keratoconus, visual acuity (VA) and methods of management for patients with keratoconus. ${ }^{9-11,14-17}$ The purpose of this study is to provide an analysis of a large cohort of keratoconus patients presenting at the University of KwaZuluNatal (UKZN) contact lens eye clinic. This clinic serves as one of the referral clinics that assesses and manages patients with keratoconus in KwaZulu-Natal. This study is important as it reports on the clinical characteristics of keratoconus patients in KwaZulu-Natal. This study thus aids in better understanding the clinical characteristics of keratoconus patients to improve knowledge of the presentation, diagnosis and management of this corneal ectasia at the UKZN contact lens clinic.

\section{Methodology}

Gatekeeper approval was also obtained from the Academic Leader at the UKZN Discipline of Optometry to access the record cards of all patients presenting at the eye clinic between January 2014 and December 2017. The study involved a retrospective review of the record cards of all patients presenting for ocular examination at the UKZN contact lens eye clinic during the study period. The study population included keratoconus patients who were presenting for assessment and fitting of contact lenses and/or follow-up. These patients were either previously diagnosed with keratoconus or were newly diagnosed during the study period with keratoconus. The ocular examination at the UKZN contact lens eye clinic includes case history, VA, corneal power measurements, anterior ocular health examination using slit lamp, spectacle refraction and contact lens evaluation (fitting and assessment). The keratoconus diagnosis was made based on the results of the ocular examination and included clinical signs associated with this corneal ectasia as determined using slit lamp examination such as corneal thinning, Munson's sign, Fleischer's ring, Vogt's striae, Rizzuti's sign and/or corneal scarring. ${ }^{2,6}$ Other clinical signs associated with the irregular corneal surface in keratoconus included the presence of scissors reflex during retinoscopy as well as distortion of the mires during keratometry and/or corneal topography.,2,6

The study entailed reviewing the clinical record cards of all keratoconus patients where data related to the study variables were collected and analysed. These variables included age at presentation to the eye clinic, the main reason for the visit, the presence of allergies, the best corrected spectacle distance VA with a Snellen acuity chart as well as the steepest and flattest corneal power measurements determined using either keratometry or corneal topography. At the UKZN eye clinic, corneal power is measured along the two principal meridians using either keratometry with a one position keratometer and/ or corneal topography with the Oculus Keratograph. Data related to the final method of management, and if keratoconus was present in only one eye (unilateral) or both eyes (bilateral), were also recorded. Keratoconus patients with ocular diseases other than keratoconus and a previous history of ocular surgery and/or trauma were excluded from the study. Moreover, keratoconus patients with any missing data in their clinical record cards, in terms of age, main reason for visit, history of allergy, surgery and/or trauma, corneal power measurements, slit lamp examination and final method of management, were also excluded from the study.

There are different classification systems that may be used to grade the severity of keratoconus such as the AmslerKrumeich classification, ABCD classification and the Collaborative Longitudinal Evaluation of Keratoconus (CLEK) study classification. In the present study, the CLEK classification system was used to grade the severity of keratoconus in each eye. According to this classification, which is based on the steepest corneal power measurement, keratoconus may be graded as either mild (corneal power less than $45 \mathrm{D}$ ), moderate (corneal power between $45 \mathrm{D}$ and $52 \mathrm{D}$ ) or severe (corneal power more than $52 \mathrm{D}$ ). The keratoconus patients' eyes were also grouped into the different levels of visual impairment, depending on the best corrected spectacle distance VA, as defined by the World Health Organization. ${ }^{18}$ Mild or no visual impairment was defined as VA of equal to or better than $6 / 18$, moderate visual impairment was defined as VA worse than $6 / 18$ but better than $6 / 60$, severe visual impairment was defined as VA worse than $6 / 60$ but better than $3 / 60$ and blindness that was defined as VA worse than $3 / 60$ but better than no light perception. ${ }^{18}$

Data were captured and analysed using the Statistical Package for Social Sciences (SPSS) version 25. Four researchers independently screened all the contact lens clinical record cards to determine whether they satisfied the criteria for the study. Only one researcher was responsible for data capturing for standardisation. To ensure accuracy of data capturing, another researcher double-checked all the data entries and any inconsistencies were resolved by cross-checking against the patient's clinical record card. The analysis comprised descriptive statistics, including mean values, standard deviations, ranges, medians, frequencies and percentages. The Shapiro-Wilk's test and histogram were used to evaluate normality of the data related to age. A probability value of less than $0.05(p<0.05)$ was considered as statistically significant.

\section{Ethical considerations}

Ethical clearance to conduct the study was obtained from the Biomedical Research and Ethics Committee at the UKZN (clearance number: BE240/18). 


\section{Results}

A total of 1210 patients were examined at the UKZN contact lens eye clinic during the study period and of these, $293(24.2 \%)$ patients had keratoconus. In this population, $209(71.3 \%)$ patients presented with bilateral keratoconus, while $84(28.7 \%)$ patients presented with unilateral keratoconus. This implies that the population comprised 502 eyes that were identified with keratoconus. However, 48 eyes of 30 patients were excluded as they failed to meet one or more of the study criteria. Consequently, the study sample consisted of 454 eyes of 263 patients with keratoconus. In the sample, $191(72.6 \%)$ and $72(27.4 \%)$ patients were found to have bilateral and unilateral keratoconus respectively. The age of the keratoconus patients ranged from 10 to 64 years with a mean and median of $25.2 \pm 9.6$ and 23 years respectively. Approximately $74 \%$ of the sample was younger than 30 years with 137 eyes from patients who were younger than 20 years and 198 eyes from patients who were aged between 20 and 30 years. Only a small proportion of 39 eyes $(8.6 \%)$ were from patients who were older than 40 years. The data on age had a non-Gaussian distribution $(p<0.05)$ and was positively skewed towards younger age (Figure 1).

The majority of the sample $(n=419,92.3 \%)$ reported refractive reasons, which included blurred and/or distorted vision, frequent change in refraction and/or progression of refractive error, as the main reason for presenting at the UKZN contact lens eye clinic. Other reasons reported for presenting at the UKZN contact lens eye clinic included cosmetic $(n=2)$, therapeutic $(n=4)$ and some patients $(n=29)$ reported two or more of the above reasons (refractive, cosmetic and/or therapeutic). There was a positive history of allergies for 189 eyes (41.6\%) in the sample. After spectacle refraction, 284 eyes $(91.6 \%)$ had no or mild visual impairment, 24 eyes $(7.7 \%)$ had moderate visual impairment and one eye each had severe visual impairment and blindness. It was not possible to determine the spectacle refraction and resulting best corrected VA in 144 eyes (18 eyes with mild keratoconus, 51 eyes with moderate keratoconus and 75 eyes with severe keratoconus).

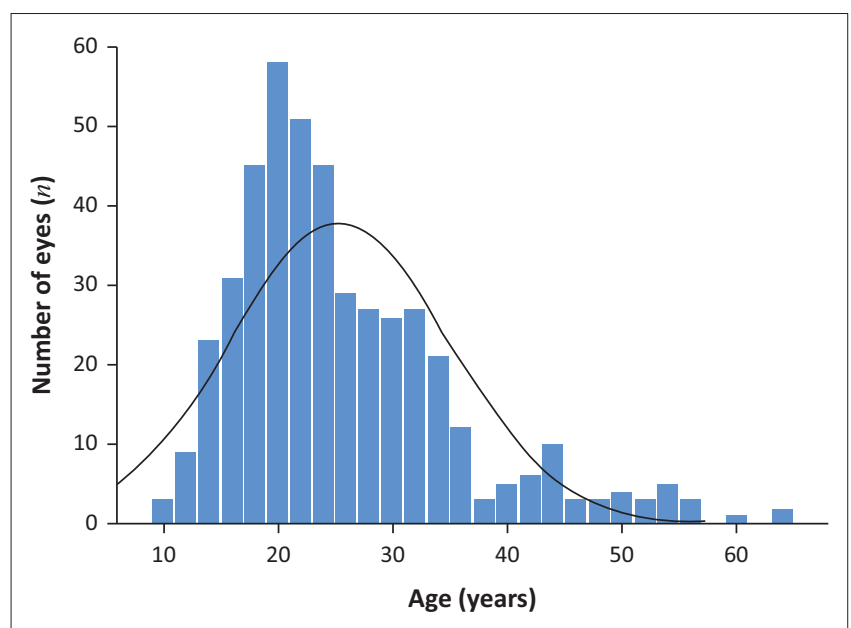

FIGURE 1: Age distribution of the study sample.
In terms of the CLEK classification of keratoconus, 41 eyes (9.0\%) had mild keratoconus, 156 (34.4\%) had moderate keratoconus and $257(56.6 \%)$ had severe keratoconus (Figure 2). Overall, the mean corneal power measurements were $54.16 \pm 7.65 \mathrm{D}$ (range: $39.50 \mathrm{D}-87.70 \mathrm{D}$, median: $52.70 \mathrm{D}$ ) and $49.15 \pm 6.97 \mathrm{D}$ (range: $34.70 \mathrm{D}-73.30 \mathrm{D}$, median: $47.90 \mathrm{D})$ along the steepest and flattest meridians respectively. The mean values and standard deviations for age and corneal variables in the three grades of keratoconus are presented in Table 1. Patients with severe keratoconus were slightly younger than those with mild and moderate keratoconus. In spite of this finding regarding the mean age of keratoconus patients, the median age was similar in these three groups (23 years, 24 years and 22 years in the mild, moderate and severe groups respectively). As the grade of keratoconus increased, the mean corneal power along the two principal meridians and amount of corneal astigmatism also increased (Table 1). In terms of management, approximately $95 \%$ of the eyes in the sample were managed with some form of optical correction with the majority $(n=418)$ being fitted with different types of rigid contact lenses (in terms of lens materials and designs) and only 13 eyes from nine patients received spectacles. Some patients received no treatment as the contact lens evaluation revealed that the eyes of these patients needed referral for surgery $(n=7)$, dry eye therapy $(n=11)$ or further investigation for other ocular pathologies $(n=5)$.

\section{Discussion}

Understanding the clinical characteristics of a disease is necessary to predict the profile of affected individuals and the need of any clinic providing care for these individuals. ${ }^{14}$ Even though keratoconus has been well documented in large-scale

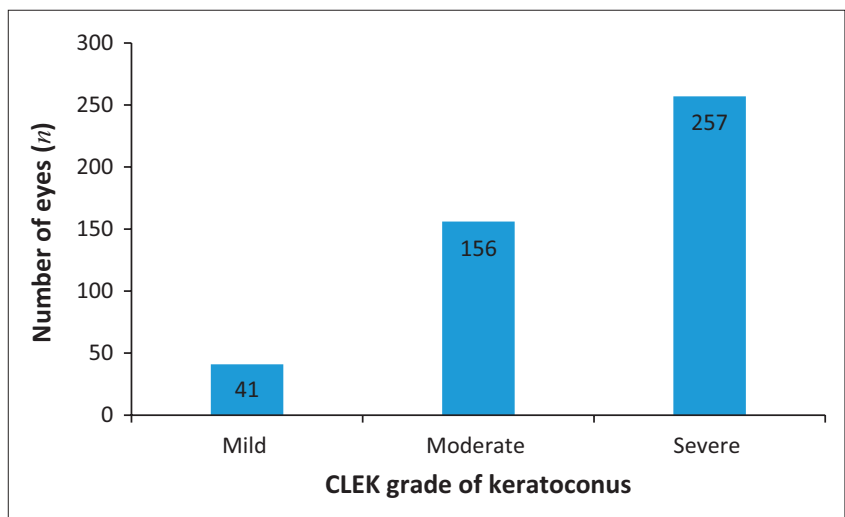

CLEK, Collaborative Longitudinal Evaluation of Keratoconus.

FIGURE 2: Number of keratoconus eyes according to the Collaborative Longitudinal Evaluation of Keratoconus classification.

TABLE 1: Mean values and standard deviations for age and corneal characteristics in the three grades of keratoconus.

\begin{tabular}{lccc}
\hline Variables & Mild & Moderate & Severe \\
\hline Number of eyes $(n)$ & 41 & 156 & 257 \\
Age (years) & $27.7 \pm 8.5$ & $25.7 \pm 10.1$ & $24.5 \pm 9.4$ \\
Steepest corneal power (D) & $43.65 \pm 1.17$ & $48.80 \pm 1.91$ & $59.53 \pm 6.14$ \\
Flattest corneal power (D) & $41.42 \pm 2.48$ & $45.10 \pm 2.85$ & $53.38 \pm 5.83$ \\
Corneal astigmatism (D) & $2.30 \pm 1.88$ & $3.77 \pm 2.43$ & $6.06 \pm 4.14$ \\
\hline
\end{tabular}

D, dioptre. 
studies conducted in the United States ${ }^{7}$ and Scotland ${ }^{5}$ as well as in hospital-based and/or clinic-based studies in Malaysia, ${ }^{15}$ Saudi Arabia, ${ }^{14}$ Singapore ${ }^{9}$ and India, ${ }^{8}$ few studies have been conducted in Africa ${ }^{16,17}$ and limited studies in South Africa specifically. Abdu et al. ${ }^{16}$ argued that knowledge of the clinical characteristics and management of keratoconus patients is important as this can inform interventions that will aid in improving the quality of life of affected individuals. This study reports on the clinical characteristics and management of keratoconus patients attending the UKZN eye clinic in KwaZulu-Natal, South Africa. Over the 4-year study period, almost $25 \%$ of all patients attending the UKZN contact lens eye clinic had keratoconus.

It is well known that keratoconus is a bilateral yet asymmetrical ocular condition where the fellow eye is at the greatest risk of progressing towards keratoconus within the first 5-6 years after the onset of this corneal ectasia. ${ }^{2,5}$ Studies have shown that the prevalence of bilateral keratoconus varies between $56.0 \%$ and $98.3 \% .^{9-11,15,17}$ In the present study, more than $70 \%$ of the patients had bilateral keratoconus and this finding is in agreement with the results of previous studies. ${ }^{10,16,17}$ For example, in the two studies conducted in Africa, Abdu et al. ${ }^{16}$ found that $77.9 \%$ of their patients had bilateral keratoconus, while Rashid et al. ${ }^{17}$ reported that $98.3 \%$ of their sample had bilateral keratoconus. Studies that have reported on keratoconus patients attending hospitals and clinics in countries outside Africa have also reported the same trend whereby the majority of patients have bilateral keratoconus. Naderan et al. ${ }^{10}$ showed that $88.3 \%$ of the keratoconus patients attending a tertiary eye-care centre over a 2-year period had bilateral keratoconus. Ameerh et al. ${ }^{11}$ reviewed the record cards of patients attending a university hospital and showed that more than $90 \%$ of their sample presented with bilateral keratoconus. An early study by Lim and $\operatorname{Vogt}^{4}$ asserted that keratoconus rarely has a unilateral presentation, and this has been shown in other studies whereby low percentage values $(4.3 \%-22.1 \%)$ of unilateral keratoconus have been reported in clinic-based and/or hospital-based studies. ${ }^{9,10,16}$ In the present study, just less than one-quarter of the sample (24.4\%) had unilateral keratoconus, which is consistent with findings in the literature. ${ }^{9,10,16}$

The mean age of patients in this study was $25.2 \pm 9.6$ years and this is slightly older than the mean age of keratoconus samples reported in studies conducted in African countries, including the Sudan ${ }^{16}(21.4 \pm 8.2$ years $)$ and Kenya ${ }^{17}$ $(21 \pm 11.1$ years). The mean age in this study is more comparable with that reported for keratoconus patients in Scotland $^{5}$ (24.1 \pm 8.9 years), Singapore ${ }^{9}$ (25.4 years) and Malaysia $^{13}$ (26.1 \pm 7.4 years). In a recent study focused on keratoconus patients in South Africa, Chetty and Rubin ${ }^{19}$ reported a mean age of $24.0 \pm 8.5$ years, which compares well with the mean age reported in the present study. In spite of these slight differences in mean age, it can be seen that patients with keratoconus usually present at clinics and/or hospitals for care mostly during their second decade of life. In the present study, the distribution of the age showed a
non-Gaussian distribution and was skewed towards younger age as has been shown for patients with keratoconus attending a specialist contact lens clinic in the study conducted by Rashid et al. ${ }^{17}$ This is an interesting finding, as despite differences in the mean age between the present study and that by Rashid et al. ${ }^{17}$ (reported above), the distribution of data related to age was similar.

In the present study, approximately three-quarters of the sample were younger than 30 years, suggesting that the patients with keratoconus who attended the UKZN contact lens eye clinic are generally adolescents and young adults. This is consistent with reports in the literature regarding the age of presentation being puberty and during the second decade of life for keratoconus patients. ${ }^{8,9}$ Mahadevan et al. ${ }^{8}$ reported that $90 \%$ of their sample of keratoconus patients, who attended a tertiary eye-care centre in South India, were aged between 10 and 30 years. Khor et al. ${ }^{9}$ reported that $61 \%$ of their keratoconus patients in a hospital-based study in Singapore were aged between 21 and 30 years. It should be noted that the mean age being described in this and the two studies conducted in South India and Singapore relates to the age of the sample when they attended the contact lens eye clinic and not the age corresponding to the onset of keratoconus. Despite this, one can infer that the age of onset of keratoconus was most likely during the teenage years or very early second decade of life for most of the patients in these samples which also corroborates with the literature related to age and onset of keratoconus. ${ }^{1-3}$ In the present study, only a small proportion of the sample (8.6\%) was older than 40 years and this is probably related to keratoconus usually stabilising in the third or fourth decade of life. ${ }^{2,4}$ This finding is not unique for this study as previous studies have also shown that relatively few keratoconus patients are diagnosed and/or present to clinics and/or hospitals after the age of 40 years. ${ }^{5,11}$ It is for this reason that Mohd-Ali et al. ${ }^{13}$ asserted that having more severe grades of keratoconus at an older age is less likely than during younger age.

The sample showed increased severity of keratoconus as most of the patients were classified with severe keratoconus (56.6\%), followed by moderate keratoconus (34.4\%) and mild keratoconus (9.0\%). This finding, in which the largest number/percentage of patients presented with severe keratoconus and the number/percentage of patients decreased as the grade of keratoconus decreased, is consistent with the trend seen in other hospital-based studies reporting on keratoconus patients. ${ }^{11,19,20}$ This finding is not surprising and may be explained by the study sample and setting comprising keratoconus patients presenting at a university clinic that serves as a referral clinic in KwaZulu-Natal. Consequently, it is likely that keratoconus patients who present at this clinic may be individuals who are no longer being fully managed by eye-care personnel (optometrists and ophthalmologists) and are therefore referred for further management. This is similar to the explanation provided by Ameerh et al. ${ }^{11}$ that keratoconus patients are often referred only when spectacles fail to provide reasonable vision. Furthermore, contact lens fitting and assessment in patients with moderate to severe 
stages of keratoconus is challenging and requires specialised equipment and expertise. Thus, it is likely that the equipment and wider range of contact lenses available at specialised contact lens clinics, such as the UKZN eye clinic, may be more suitable to provide for the needs of patients with more advanced stages of keratoconus. Previous studies have reported the same trend of a higher proportion of patients with severe keratoconus attending specialised contact lens clinics and hospitals. ${ }^{6,8,17}$ Saini et al., ${ }^{6}$ who reported on keratoconus patients attending a tertiary eye-care facility and used the same keratoconus classification system as the present study, reported that $67.2 \%$ and $32.8 \%$ of their patients' eyes had severe and moderate keratoconus respectively. Mahadevan et al. ${ }^{8}$ also found that the majority of their keratoconus patients from a tertiary eye-care centre $(42 \%)$ had advanced keratoconus defined as a corneal curvature value greater than $52 \mathrm{D}$. Taken together, the findings of this and previous studies are probably biased as they contain an over-representation of patients with severe keratoconus because of the data being extracted from specialised contact lens clinics and hospitals to which these specific patients are most likely referred.

Several clinic-based and hospital-based studies have reported that contact lenses (soft and rigid) have been used as the primary method of correction for their samples of keratoconus patients. ${ }^{4,9,15,16,17,21}$ This may be the case as contact lenses allow for good visual outcomes in keratoconus patients and delay the need for surgery such as penetrating keratoplasty. ${ }^{20}$ In the CLEK study, $74 \%$ of the patients were prescribed with either soft or rigid contact lenses, while only $16 \%$ received spectacles as the primary method of vision correction..$^{21} \mathrm{~A}$ more recent study done by Rashid et al. ${ }^{17}$ showed that $98 \%$ of their sample was managed with spectacles or rigid contact lenses with only $2 \%$ receiving no treatment. Fathima et al. ${ }^{20}$ conducted a retrospective analysis of keratoconus patients attending a hospital in India and reported that $79.5 \%$ of these individuals were managed with rigid contact lenses. A similar trend was observed in this study where the most common method of management was different types of rigid contact lenses in terms of materials and designs. This finding is most likely explained by the majority of the sample having moderate to severe keratoconus where prescription of spectacles or soft contact lenses may not allow for optimal vision correction because of the irregular corneal surface. ${ }^{3}$ Consequently, rigid contact lenses may be preferred as they result in better vision correction by providing a more uniform optical surface for refraction of light rays. ${ }^{2,13}$ In the UKZN contact lens clinic, different materials and designs of rigid contact lenses are used to fit the keratoconus patients using three fitting philosophies (three-point touch, apical clearance and apical bearing). Khor et al. ${ }^{9}$ speculated that the reliance on the use of contact lenses is due to the severity of keratoconus that patients present with when they are being assessed and managed in specialised contact lens clinics. This is probable as it is well known that moderate to severe stages of keratoconus are difficult to manage with the use of spectacles. ${ }^{3}$ Overall, only a few patients were prescribed with spectacles as the method of management which is in agreement with the findings of the CLEK study. ${ }^{21}$ Some of the possible reasons for keratoconus patients opting for spectacles could be that they may have had early stages of this corneal ectasia and/or may not be able to tolerate rigid contact lenses. ${ }^{15}$

The findings related to reasons for reporting to the clinic and history of allergies in this study are consistent with literature reports. More than $90 \%$ of the sample reported refractive reasons as the main reason for presenting at the clinic and this finding is in agreement with the reports from previous studies. ${ }^{4,8,9,17}$ In this and previous studies, refractive reasons were defined as poor vision (blur and/or distorted) not adequately corrected by spectacles, progression of refractive error and/or frequent change in spectacles. ${ }^{4}$ The complaint of refractive reasons is most likely explained by a large proportion of the keratoconus patients' eyes $(n=257)$ being classified with severe keratoconus, which is associated with poorer visual function, than mild and/or moderate keratoconus. ${ }^{20}$ More than $40 \%$ of the sample reported a positive history of allergies which is consistent with the results of the CLEK study where approximately $53 \%$ of their sample reported positively for allergies. ${ }^{7}$ Although there are reports of a possible association between the presence of allergic conditions and keratoconus, ${ }^{22}$ the exact mechanism of these allergic conditions on the pathogenesis of keratoconus is still unknown. It is for this reason that, rather than the presence of allergic conditions only, the behavioural pattern of excessive eye rubbing in individuals with a history of allergic conditions has been associated with the development and progression of keratoconus..$^{5,13}$

The comparison of age and corneal clinical characteristics among the three grades of keratoconus patients revealed interesting findings. In terms of age, the severe keratoconus patients were slightly younger than the patients with moderate and mild keratoconus, which is consistent with the results of a previous study. ${ }^{6}$ When describing a possible relation between age and severity of keratoconus, Naderan et al. ${ }^{10}$ observed that patients who had an earlier onset of keratoconus presented with more severe grades and this may be because of the progressive nature of this corneal ectasia. The findings, that corneal power in the two principal meridians and amount of corneal astigmatism increased as the grade of keratoconus increased, are not unexpected and are probably attributed to keratoconus being associated with progressive structural changes in terms of corneal thinning and protrusion. ${ }^{3}$

Even though an earlier study suggested that keratoconus has no gender predilection, ${ }^{1}$ there are contradictory reports in the literature regarding which gender group is more affected by keratoconus. The majority of studies have shown that there are gender differences in the proportion of keratoconus with more males than females being affected by this corneal ectasia. ${ }^{8,9,11,15,16,17}$ However, Saini et al., ${ }^{6}$ who reported on keratoconus patients attending a tertiary eye-care facility in North India, found that there were slightly more females than males in their sample. Despite these inconsistent results, 
it has been shown that there are no gender differences for the mean corneal power along the two principal meridians and severity of keratoconus. ${ }^{13}$ In the present study, it was not possible to report on gender and its influence on keratoconus as the current clinical record cards at the UKZN contact lens eye clinic do not allow for recording of this demographic characteristic. It should be noted that this limitation of the current recording system has been incorporated into the revised computerised clinical record cards that are going to be implemented at the UKZN eye clinic. Moreover, it was not possible to report on the prevalence and/or incidence of keratoconus in the sample or different gender groups as this study only included the record cards of keratoconus patients that were presenting at the UKZN contact lens clinic for assessment and fitting of contact lenses and/or follow-up.

Limitations of this study include its retrospective design and that the contact lens ocular examinations were performed by different clinicians at the UKZN contact lens eye clinic. As the study site was an eye clinic in a university, it may not necessarily describe the keratoconus patients presenting at private and/or public optometric practices for routine assessment and management. Moreover, the inherent selection bias in this study because of the study site should be considered as the keratoconus patients included in the study were only those that presented for assessment and management at this specific eye clinic in KwaZulu-Natal. Consequently, it is possible that some of the keratoconus patients would have been referred for surgery before being referred to the UKZN contact lens clinic and that some patients with mild and/or incipient keratoconus would be under-represented in the sample as they are being managed by other optometric personnel. As a consequence of the study design, it was not possible to determine the incidence, prevalence and age of onset of keratoconus. Despite the sample including a large number of keratoconus patients at the UKZN contact lens eye clinic, it is recommended that future prospective studies be undertaken at other sites within KwaZulu-Natal to better understand the nature, clinical presentation and management of this corneal ectasia.

\section{Conclusion}

To the best of the researchers' knowledge, this is the first study to report on the clinical characteristics of keratoconus patients at the UKZN contact lens eye clinic. The results of this study show that keratoconus patients who present at an early age have a more severe grade of this corneal ectasia and are most commonly managed with the use of rigid contact lenses. These findings should be considered for keratoconus screening, diagnosis and treatment programmes in KwaZulu-Natal to improve the quality of life of affected individuals. This is because the early detection and management of keratoconus can help to enhance the quality of life of affected individuals.

\section{Acknowledgements}

The authors thank Nyika Mtemeri for assistance with statistical analysis.

\section{Competing interests}

The authors declare that they have no financial or personal relationship(s) that may have inappropriately influenced them in writing this article.

\section{Authors' contributions}

S.R. was the study leader. N.G. and N.R. provided feedback on the study design. N. Madlala, N. Memela, S.N., N. Shabalala, N. Simjee and S.R. performed data collection. N.R. and N.G. wrote the manuscript.

\section{Funding information}

This research received no specific grant from any funding agency in the public, commercial or non-for-profit sectors.

\section{Data availability statement}

New and original data were collected, analysed and presented for this study.

\section{Disclaimer}

The views and opinions expressed in this article are those of the authors and do not necessarily reflect the official policy or position of any affiliated agency of the authors.

\section{References}

1. Rabinowitz YS. Keratoconus. Surv Ophthalmol. 1998;42(4):297-319. https://doi org/10.1016/S0039-6257(97)00119-7

2. Kanski JJ. Clinical ophthalmology: A systematic approach. 6th ed. London: Butterworth-Heinmann, 2007; p. 288-290.

3. Romero-Jiménez $M$, Santodomingo-Rubido J, Wolffsohn JS. Keratoconus: A review. Cont Lens Anterior Eye. 2010;33(4):157-166. https://doi.org/10.1016/j. clae.2010.04.006

4. Lim N, Vogt U. Characteristics and functional outcomes of 130 patients with keratoconus attending a specialist contact lens clinic. Eye. 2002;16(1):54-59. https://doi.org/10.1038/sj.eye.6700061

5. Weed KH, MacEwen CJ, Giles T, Low J, McGhee CNJ. The Dundee University Scottish Keratoconus Study: Demographics, corneal signs, associated diseases, and eye rubbing. Eye. 2008;22(4):534-541. https://doi.org/10.1038/sj.eye. and eye
6702692

6. Saini JS, Saroha V, Singh P, Sukhija JS, Jain AK. Keratoconus in Asian eyes at a tertiary eye care facility. Clin Exp Optom. 2004;87(2):97-101. https://doi.org/ 10.1111/j.1444-0938.2004.tb03155.x

7. Zadnik K, Barr JT, Edrington TB, et al. Baseline findings in the Collaborative Longitudinal Evaluation of Keratoconus (CLEK) study. Invest Ophthalmol Vis Sci. 1998;39(13):2537-2546.

8. Mahadevan R, Arumugam AO, Arunachalam V, Kumaresan B. Keratoconus A review from a tertiary eye-care centre. J Optom. 2009;2(4):166-172. https:// doi.org/10.3921/joptom.2009.166

9. Khor WB, Wei RH, Lim L, Chan CM, Tan DTH. Keratoconus in Asians: Demographics, clinical characteristics and visual function in a hospital-based population. Clin Exp Ophthalmol. 2011;39(4):299-307. https://doi.org/10.1111/j.1442-9071. 2010.02458.x

10. Naderan M, Shoar S, Rezagholizadeh F, Zolfaghari M, Naderan M. Characteristics and associations of keratoconus patients. Cont Lens Anterior Eye. 2015;38(3): 199-205. https://doi.org/10.1016/j.clae.2015.01.008

11. Ameerh MAA, Al Refai RM, Al Bdour MD. Keratoconus patients at Jordan University hospital: A descriptive study. Clin Ophthalmol. 2012;6:1895-1899. https://doi.org/10.2147/OPTH.S38287

12. Pearson AR, Soneji B, Sarvananthan N, Sandford-Smith JH. Does ethnic origin influence the incidence or severity of keratoconus? Eye. 2000;14(4):625-628. https://doi.org/10.1038/eye.2000.154

13. Mohd-Ali B, Abdu M, Das S, Mohidin N. Ethnicity related to keratoconus: A study with clinical implications. Int Med J. 2011;18(3):237-240.

14. Assiri AA, Yousuf BI, Quantock AJ, Murphy PJ. Incidence and severity of keratoconus in Asir province, Saudi Arabia. Br J Ophthalmol. 2005;89(11): 1403-1406. https://doi.org/10.1136/bjo.2005.074955 
15. Mohd-Ali B, Abdu M, Yaw CY, Mohidin N. Clinical characteristics of keratoconus patients in Malaysia: A review from a cornea specialist centre. J Optom. 2012;5(1):38-42. https://doi.org/10.1016/j.optom.2012.01.002

16. Abdu M, Binnawi KH, Elmadina AEM, Hassan R. Clinical profile of keratoconus patients in Sudan. Sudanese J Ophthalmol. 2016;8(1):20-25. https://doi.org/ 10.4103/1858-540X.184235

17. Rashid ZA, Millodot M, Evans KSE. Characteristics of keratoconic patients attending a specialist contact lens clinic in Kenya. Middle East Afr J Ophthalmol. 2016;23(4):283-287. https://doi.org/10.4103/0974-9233.194074

18. World Health Organization (WHO). International statistical classification of diseases and related health problems, 10th revision (ICD-10) [homepage on the Internet]. 2016 [cited 2019 Jul 24]. Available from: https://icd.who.int/browse10/ 2015/en\#/H54.
19. Chetty E, Rubin A. Preliminary demographics for patients with keratoconus attending a university-based clinic in Johannesburg South Africa. Afr Vision Eye Health. 2019;78(1):1-5. https://doi.org/10.4102/aveh.v78i1.472

20. Fathima T, Acharya MC, Mathur U, Barua P. Demographic profile and visual rehabilitation of patients with keratoconus attending contact lens clinic at a tertiary eye care centre. Cont Lens Anterior Eye. 2010;33(1):19-22. https://doi. org/10.1016/j.clae.2009.09.004

21. Wagner H, Barr JT, Zadnik K. Collaborative Longitudinal Evaluation of Keratoconus (CLEK) study: Methods and findings to date. Cont Lens Anterior Eye. 2007;30(4): 223-232. https://doi.org/10.1016/j.clae.2007.03.001

22. Shneor E, Millodot M, Blumberg S, Ortenberg I, Behrman S, Gordon-Shaag A. Characteristics of 244 patients with keratoconus seen in an optometric contact len practice. Clin Exp Optom. 2013;96(2):219-224. https://doi.org/10.1111/cxo.12005 